\title{
Modeling of Dimensionally Graded Magnetoelectric Energy Harvester
}

\author{
R.V. Petrov ${ }^{1}$, V.M. Petrov ${ }^{1}$, M.I. Bichurin ${ }^{1}$, Y.Zhou ${ }^{2}$ and S. Priya ${ }^{2}$
}

${ }^{1}$ Department of Design and Technology of Radio equipment, Novgorod State University, Veliky Novgorod, Russia; ${ }^{2}$ Virginia Polytechnic Institute and State University, Bio-Inspired Materials and Devices Laboratory, Center for Energy Harvesting Materials and Systems, Blacksburg, VA 24061, USA

E-mail: Roman.Petrov@novsu.ru

Abstract - The magnetoelectric behaviour of a dual-phase dimensionally graded magnetostrictivepiezoelectric composite is modeled in this article. The cantilever is formed by piezoelectric macro-fiber composite bonded to a Ni cantilever. Theoretical estimates show a large magnetoelectric voltage coefficient of $100 \mathrm{~V} /(\mathrm{cm} \mathrm{Oe})$ at electromechanical resonance frequency. An additive effect was realized when an acceleration and magnetic field was applied to the structure simultaneously. Applied magnetic field of 10 Oe and shaker acceleration of $0.02 \mathrm{~g}$ induce the approximately equal output voltage of $20-35 \mathrm{~V}$.

\section{Keywords-Magnetoelectric coupling; bending mode; energy harvesting}

\section{Introduction}

Magnetoelectrics are materials that become electrically polarized in an applied magnetic field and magnetized in an applied electric field. Magnetoelectrics are potentially applicable in magnetic field sensors and magnetoelectric transducers due to their intrinsic effect of conversion between magnetic and electric fields. Single-phase materials cannot be used in any application since they have very weak magnetoelectric (ME) coupling and their operating temperatures are usually below the room temperature. It is reasonable to develop two-phase composite materials by using the product property of the magnetostrictive effect in a magnetostrictive phase and the piezoelectric effect in a piezoelectric phase [1]. This is enabled since a magnetic field applied to such a magnetoelectric composite induces a strain in the magnetostrictive phase that is transferred into the piezoelectric phase to generate a piezoelectric voltage or charge proportional to the applied magnetic field. 
Piezoelectric cantilever based structures are widely used in vibration energy harvesters as they show low operating frequency that can be tuned by using the tip mass [2, 3]. Piezoelectric and ME coupling in ME composites result in a summation of output voltages induced by both mechanical and magnetic excitations. This suggests using the ME composites including the structures with dual mode operation regime in energy-harvesting applications [4-8]. The energy collection and conversion effectiveness are expected to be enhanced under magnetic and mechanical excitation. Because the ME effect in composites is due to mechanically coupled piezoelectric and magnetostrictive subsystems, it sharply increases in the vicinity of the electromechanical resonance (EMR) frequency [1].

We report a theoretical model for a magnetoelectric energy harvesting structure that can simultaneously scavenge magnetic and vibration energy. The structure considered is designed by combining a Ni unimorph and bilayer of piezoelectric microfiber composite (MFC) and $\mathrm{Ni}$ in a cantilever configuration as in Fig. 1 [9]. The MFC is extremely flexible, allowing it to be bonded to structures that have curved surface without fear of accidental breakage or additional surface treatmentas is the case with monolithic piezoceramic materials. Additionally the MFC uses interdigitated electrodes that capitalize on the higher piezoelectric coupling coefficient $d_{33}$ which enables producing the higher forces and strain than typical monolithic piezoceramic materials. $\mathrm{Ni}$ has been incorporated into $\mathrm{ME}$ laminate composites as the magnetostrictive phase. The Ni beam plays two important roles in this configuration: (i) a magnetostrictive phase in the ME laminate, (ii) a magnetic-field-active cantilever for the piezoelectric bender [9].

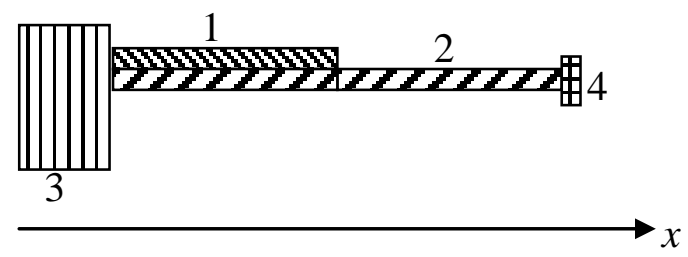

Fig. 1 In-series connected bilayer of MFC and Ni (1) and Ni unimorph (2) rigidly clamped by shaker (3) at $\mathrm{x}=0$ and loaded by tip mass $(4)$ at $\mathrm{x}=\mathrm{L}+\mathrm{L}_{1}$ 


\section{Theoretical Modeling}

An in-plane bias field is assumed to be applied to magnetostrictive component to provide the strong piezomagnetic coupling and avoid the demagnetizing field. The thickness of the plate is assumed to be small compared to remaining dimensions. Moreover, the plate width is assumed small compared to its length. In that case, we can consider only one component of strain and stress tensors in the EMR region. Theoretical modeling rests on the assumption that the sample undergoes the superposition of axial and bending strain [1]. These strains can be calculated by using the elastostatic/elastodynamic equations and then they can be expressed in terms of stress components with the aid of elasticity law. To obtain the expression for the ME voltage coefficient, we substitute the found stress components into the open circuit condition.

We consider a model for ME coupling at EMR. A key drawback for ME effect at longitudinal modes is that the frequencies are quite high, on the order of hundreds of $\mathrm{kHz}$, for nominal sample dimensions. The eddy current losses for the magnetostrictive phase can be quite high at such frequencies resulting in an inefficient magnetoelectric energy conversion. In order to reduce the operating frequency, one can use bending modes of the composite. The frequency of applied ac field is expected to be much lower compared to longitudinal acoustic modes. In this work, we focus our attention on theoretical modeling of ME effects at bending modes. We assume the sample in the $(x, y)$ plane i.e. $(1,2)$ plane with its thickness along the $z$-axis (3 axis).

Theoretical modeling described here is based on the equations for the strain, electric displacement and magnetic induction of piezoelectric and magnetostrictive phases:

$$
\begin{aligned}
& { }^{\mathrm{p}} \mathrm{S}_{\mathrm{i}}={ }^{\mathrm{p}} \mathrm{S}_{\mathrm{ij}}{ }^{\mathrm{p}} \mathrm{T}_{\mathrm{j}}+{ }^{\mathrm{p}} \mathrm{d}_{\mathrm{ki}}{ }^{\mathrm{p}} \mathrm{E}_{\mathrm{k}} ; \\
& { }^{\mathrm{p}} \mathrm{D}_{\mathrm{k}}={ }^{\mathrm{p}} \mathrm{d}_{\mathrm{ki}}{ }^{\mathrm{p}} \mathrm{T}_{\mathrm{i}}+{ }^{\mathrm{p}} \varepsilon_{\mathrm{kn}}{ }^{\mathrm{p}} \mathrm{E}_{\mathrm{n}} ; \\
& { }^{\mathrm{m}} \mathrm{S}_{\mathrm{i}}={ }^{\mathrm{m}} \mathrm{S}_{\mathrm{ij}}{ }^{\mathrm{m}} \mathrm{T}_{\mathrm{j}}+{ }^{\mathrm{m}} \mathrm{q}_{\mathrm{ki}}{ }^{\mathrm{m}} \mathrm{H}_{\mathrm{k}} ; \\
& { }^{\mathrm{m}} \mathrm{B}_{\mathrm{k}}={ }^{\mathrm{m}} \mathrm{q}_{\mathrm{ki}}{ }^{\mathrm{m}} \mathrm{T}_{\mathrm{i}}+{ }^{\mathrm{m}} \mu_{\mathrm{kn}}{ }^{\mathrm{m}} \mathrm{H}_{\mathrm{n}} ;
\end{aligned}
$$

where $S_{i}$ and $T_{j}$ are strain and stress tensor components, $E_{k}$ and $D_{k}$ are the vector components of electric field and electric displacement, $H_{k}$ and $B_{k}$ are the vector components of magnetic field and magnetic induction, $s_{i j}, q_{k i}$ and $d_{k i}$ are compliance, piezomagnetic and piezoelectric coefficients, $\varepsilon_{k n}$ is the permittivity 
matrix, and $\mu_{k n}$ is the permeability matrix. The superscripts $p$ and $m$ correspond to piezoelectric and piezomagnetic layers, respectively. We assume the symmetry of piezoelectric to be $\infty \mathrm{m}$ and the magnetic to be cubic.

The small-amplitude bending oscillations of the sample are governed by the following equations for MFC -Ni bilayer and Ni unimorph:

$$
\begin{aligned}
& \nabla^{2} \nabla^{2} w+\frac{\rho t}{D} \frac{\partial^{2} w}{\partial \tau^{2}}=0 \\
& \nabla^{2} \nabla^{2} w_{1}+\frac{{ }^{m} \rho}{{ }^{m} D} \frac{{ }^{m}}{\partial} \frac{\partial^{2} w_{1}}{\partial \tau^{2}}=0
\end{aligned}
$$

where $\nabla^{2} \nabla^{2}$ is biharmonic operator, $w$ and $w_{l}$ are the deflections (displacements in z-direction) for MFC Ni bilayer and Ni unimorph, $t$ and $\rho$ are thickness and average density of bilayer, $t={ }^{p} t+{ }^{m} t, \rho=\left(\rho^{p} t+{ }^{m} \rho^{m} t\right) / t$, with ${ }^{p} \rho,{ }^{m} \rho,{ }^{p} t$ and ${ }^{m} t$ are densities and thicknesses of piezoelectric and magnetic layers, respectively, $\tau$ is the time, and $D$ and ${ }^{m} D$ is cylindrical stiffnesses for bilayer and Ni unimorph. The thickness of the sample is assumed to be small compared to other dimensions and its width small compared to its length. In that case, we can consider only one component of strain and stress. The MFC -Ni bilayer with length $L$ is assumed to be joined at $x=L$ to a $N i$ plate with length $L_{1}$.

The induced voltage is calculated at open circuit condition $\int_{0}^{L}{ }_{0}^{p} D_{1} d x=0$ with ${ }^{p} D_{1}$ denoting the electric induction. Using Eqs. 1, the ME voltage coefficient $\alpha_{E}=E_{1} / H_{1}$ can be found as

$$
\alpha_{E}=-\int_{A} \frac{{ }^{p} d_{33}^{p} T_{1}}{L b H_{1}^{p} \varepsilon_{33}} d S,
$$

where $A$ is the cross section of piezoelectric layer in $(x, z)$ plane and $b$ is the width of the sample.

The expression for stress component ${ }^{p} T_{1}$ in Eq. (3) was found from the law of elasticity (Eq. 1) for piezoelectric layer. The strains in Eq. 1 can be expressed in terms of deflection as ${ }^{p} S_{1}=-z_{1} \frac{\partial^{2} w}{\partial x^{2}}$, where $z_{1}$ is measured from the middle plane of the sample.

In what follows we restrict ourselves to the case of harmonic oscillations. Since solutions of Eqs. 2 depend on sample's end conditions, we consider two types of end fixity conditions.

\section{(i) MFC-Ni cantilever joined to Ni plate with no tip mass}

We use the boundary conditions for the bilayer with length $L$ rigidly clamped at one end (at $x=0)$ and 
joined to a $N i$ plate with length $L_{l}$. For this case, the boundary conditions take on form:

$$
M_{y}=0 \text { and } V_{y}=0 \text { at } x=L+L_{1} ;
$$

where $M_{y}$ is the torque moment relative to $y$-axis produced by internal stresses per unite width and $V_{y}$ is the transverse force per unite width.

The final expression for ME voltage coefficient is not cited here because of space limitation and we restrict ourselves to point-by-point computation of the ME coefficient's frequency dependence. The calculation procedure is as follows: the general solutions of Eqs. 2 is written as $w(x)=C_{1} \cos k x+$ $C_{2} \cosh k x+C_{3} \sin k x+C_{4} \sinh k x$ and $w_{1}(x)=C_{5} \cos k_{1} x+C_{6} \cosh k_{1} x+C_{7} \sin k_{1} x+C_{8} \sinh k_{1} x$ where $C_{i}$ are the constants of integration, $k=\sqrt[4]{\omega^{2} \frac{{ }^{p} \rho^{p} t+{ }^{m} \rho^{m} t}{D}}$, and $k_{1}=\sqrt[4]{\omega^{2} \frac{\rho^{1} t}{{ }^{1} D}}$ with $D,{ }^{1} D,{ }^{1} \rho$, and ${ }^{1} \mathrm{t}$ denoting cylindrical stiffness for MFC -Ni cantilever, cylindrical stiffness, density, and thickness for Ni plate, correspondingly. Next for the selected values of frequency, the constants of integration are found from boundary conditions and substituted into expressions for deflections taking into account the material parameters of the structure. Finally, the strain and stress components are calculated and substituted into Eq. 3 to find the frequency dependence of ME voltage coefficient and output voltage point by point.

The transducer can be excited by both ac magnetic field generated by the Helmholtz coil and a mechanical oscillation generated by the shaker at $x=0$. Fig. 2 shows the frequency dependence of induced voltage by applied ac magnetic field and shaker acceleration for the cantilever with no tip mass. The following parameters are used for estimates: $L=L_{l}=40 \mathrm{~mm}, b=5 \mathrm{~mm},{ }^{p} t=0.2 \mathrm{~mm},{ }^{m} t={ }^{l} t=0.2 \mathrm{~mm},{ }^{p} Y=30$ $\mathrm{GPa},{ }^{m} Y=2000 \mathrm{GPa},{ }^{m} q_{11}=1.1 \cdot 10^{-9} \mathrm{~m} / \mathrm{A},{ }^{p} d_{33}=0.4 \cdot 10^{-9} \mathrm{~m} / \mathrm{V},{ }^{p} \rho=5.44 \mathrm{~g} / \mathrm{cm}^{3},{ }^{m} \rho=8.9 \mathrm{~g} / \mathrm{cm}^{3},{ }^{p} \varepsilon_{33} / \varepsilon_{0}=1750$. Resonance losses are taken into account by a complex frequency $\omega+i \omega^{\prime}$ with $\omega^{\prime} / \omega=10^{-2}$. 

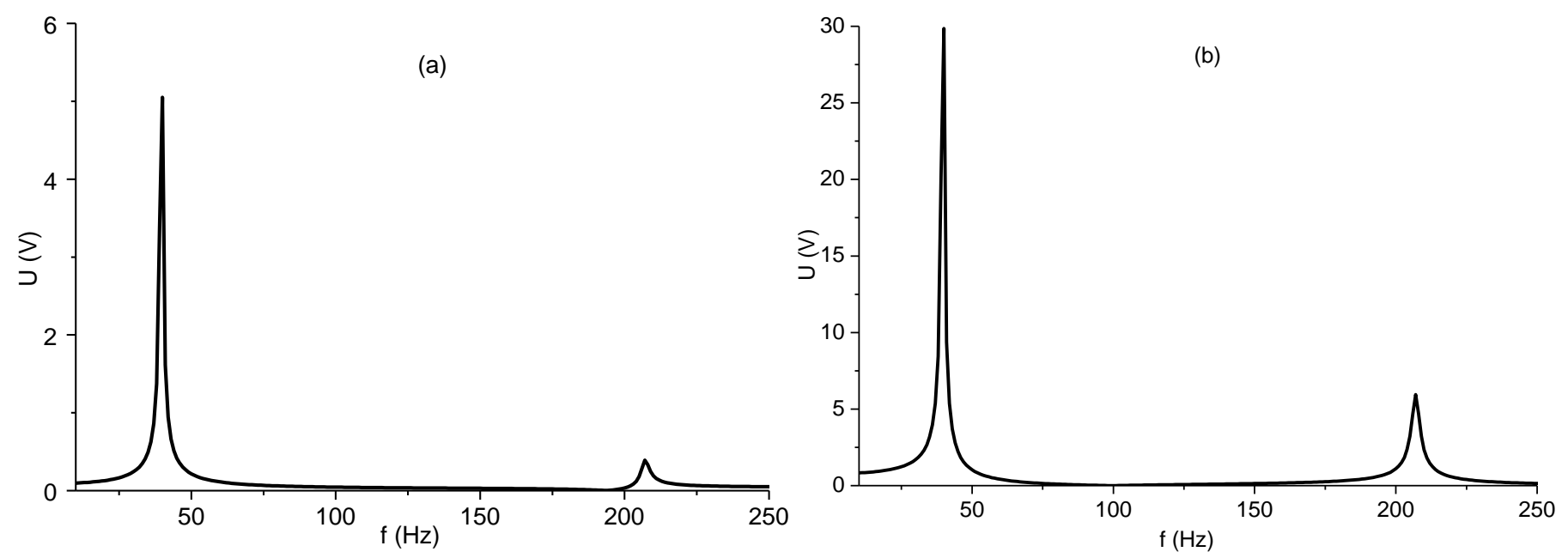

Fig. 2 Frequency dependence of open circuit voltage for the cantilever with no tip mass for applied ac magnetic field of $10 \mathrm{Oe}$ (a) and shaker acceleration of $0.02 \mathrm{~g}$ (b) with $\mathrm{g}$ denoting the gravitational acceleration.

The frequency dependence of output voltage in Fig. 2 shows that the ME effect sharply increases in the bending resonance region.

\section{(ii) MFC -Ni cantilever joined to Ni plate with tip mass}

A lower operating frequency and stronger ME coupling can be obtained by adding the tip mass. Boundary conditions for the $N i$ plate take into account the tip mass and can be written as:

$$
M_{y}=\partial w / \partial x I \omega^{2} / b \text { and } V_{y}=-m w \omega^{2} / b \text { at } x=L+L_{l},
$$

where $m$ and $I$ are mass and moment of inertia of tip mass with respect to axis that is positioned in the middle plane along $y$ axis, correspondingly, and $b$ is the sample width.

The frequency dependence of ME voltage coefficient for a MFC -Ni cantilever joined to Ni plate with tip mass of $1.1 \mathrm{~g}$ is shown in Fig. 3. For simplicity, we neglect the influence of the moment of inertia of tip mass. 

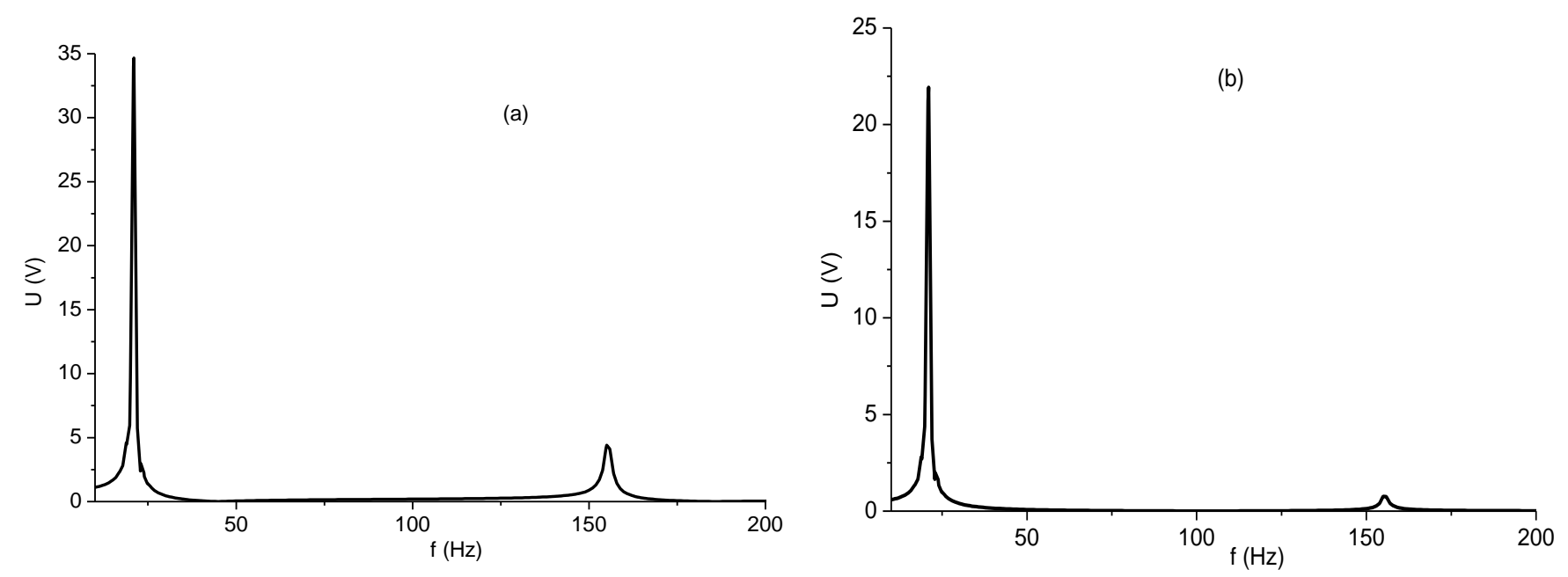

Fig. 3 Frequency dependence of open circuit voltage for the cantilever with tip mass of $1.1 \mathrm{~g}$ for applied ac magnetic field of $10 \mathrm{Oe}$ (a) and shaker axeleration of $0.02 \mathrm{~g}$ (b) with g denoting the gravitational acceleration.

To enable the summation effect for both mechanical and magnetic energies, a bilayer of MFC and Ni is considered with magnetic and mechanical excitation. The stress and magnetic field energy could be converted into electric charges in the piezoelectric layer through a direct piezoelectric effect and ME effect, correspondingly. This combination should enhance energy collection. It should be noted that vibrational sources of energy production are especially attractive because the associated harvesters can be scaled to incredibly small sizes and do not need the constant addition of fuel.

In contrast to the case considered above, the boundary conditions for the bilayer should take into account the rigid clamping and acceleration $\boldsymbol{a}$ induced by shaker along $\mathrm{z}$-axis at $x=0$. So we have

$$
\partial w / \partial x=0 \text { and } \partial^{2} w / \partial \tau^{2}=a \text { at } x=0 .
$$

The frequency dependence of output voltage for mechanical excitation is shown in Fig. 3. The acceleration of sample at clamped end is assumed frequency-independent.

The frequency dependence of output voltage under magnetic excitation, acceleration of clamped end and simultaneously applied magnetic field and shaker acceleration is shown in Fig. 4. 


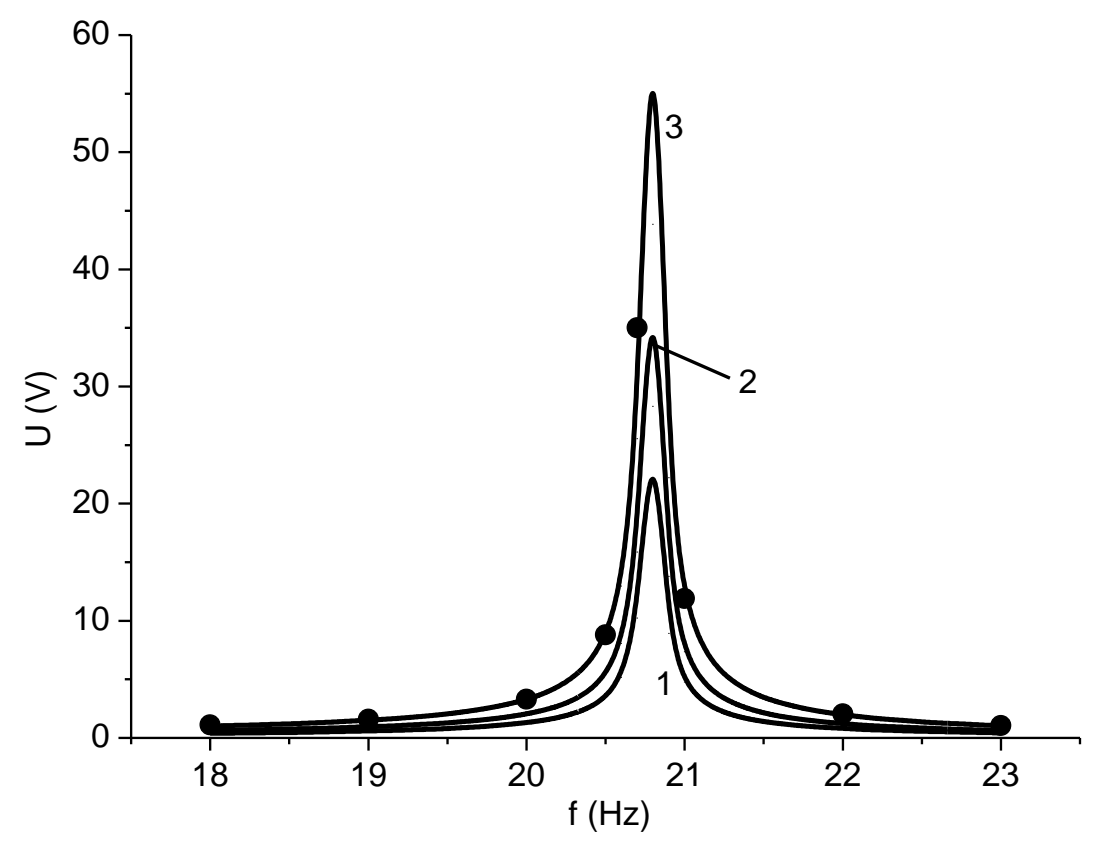

Fig. 4 Frequency dependence of output voltage in the region of first resonance frequency for applied magnetic field of 10 Oe (curve 1), shaker acceleration of $0.02 \mathrm{~g}$ (curve 2), and simultaneously applied magnetic field of $10 \mathrm{Oe}$ and shaker acceleration of $0.02 \mathrm{~g}$ (curve 3). Here g denotes the gravitational acceleration. Points correspond to the data for simultaneously applied magnetic field and shaker acceleration.

Fig. 4 gives evidence of summation effect for magnetic and mechanical excitations. Magnetic field of $10 \mathrm{Oe}$ and acceleration of shaker of $0.02 \mathrm{~g}$ ( $\mathrm{g}$ is gravitational acceleration) result in approximately equal output voltage.

To investigate the summation effect experimentaly, the transducer was simultaneously excited by an ac magnetic field generated by the Helmholtz coil and a mechanical oscillation generated by the shaker. When placed in an ac magnetic field, the magnetostrictive layer can elongate or contract, thereby, straining the piezoelectric layer that results in output voltage across the piezoelectric layer. In-plane bias magnetic field of 180 Oe was applied to the magnetic layer to provide the maximal piezomagnetic coefficient and ME output. Under the external vibration, oscillation of the cantilever can directly create strain in the piezoelectric layer and therefore produce electrical voltage. The net effect of acoustic and magnetic ac 
excitations depends on the phase difference. It is clear that the maximal total effect is obtained for zero phase difference between acoustic and magnetic ac excitations and the minimum one must be obtained when magnetic field and shaker vibrations are opposite in phase. Thus we studied the maximum effect for acoustic excitation in-phase with magnetic one. The ME response was measured for in-plane longitudinal fields orientation (longitudinally magnetized and longitudinally poled sample) with sample located in the center of the Helmholtz coil. Our measuring setup is similar to the setup described recently [4]. This setup enables one to excite the ME transducer by ac magnetic field and a mechanical oscillation generated by the shaker. The induced ME voltage was measured using a lock-in amplifier. Mechanical excitation was generated by the shaker using a function generator. The measured frequency dependence of output voltage for simultaneously applied magnetic field of 10 Oe and shaker acceleration of $0.02 \mathrm{~g}$ is shown in Fig. 3.

Data in Fig. 4 show a satisfactiry agreement between the theoretical estimates and data. The peak value of induced voltage is obtained at resonance frequency.

The most important characteristic of an energy harvester is the generated electrical power, $P=U^{2} / 2 R$ with $U$ and $R$ denoting the voltage output and external load. To estimate the generated electrical power, the open circuit condition is replaced by $\int_{0}^{L} \frac{\partial^{p} D_{1}}{\partial t} d x=\frac{U}{b R}$ where $b$ is the sample width. The vibration induced maximum electrical power output equals $60 \mu \mathrm{W}$ at a matching load of $4 \mathrm{M} \Omega$ and at $\mathrm{f}=21 \mathrm{~Hz}$ and shaker acceleration of $0.02 \mathrm{~g}$. This power is high compared to other ME bender. It should be noted that the power output increases with increasing acceleration. These results clearly demonstrate that a high output power can be achieved by using the dimensionally graded system with tip mass.

\section{Conclusions}

In this paper, a theoretical study of the $\mathrm{ME}$ effect in a two-phase dimensionally graded magnetostrictive-piezoelectric structure. The composite is formed by the series-connected magnetostrictivepiezoelectric bilayer and a magnetic plate. Theory predicts a large magnetoelectric voltage coefficient of 
$100 \mathrm{~V} /(\mathrm{cm} \mathrm{Oe})$ at electromechanical resonance frequency. We show that the generated electric energy is sum effect from magnetoelectric and piezoelectric contributions. It is shown that the applied magnetic field of $10 \mathrm{Oe}$ and acceleration of the fixed end of the sample of $0.02 \mathrm{~g}$ lead to induced voltage of 20 to $35 \mathrm{~V}$. Data are in a satisfactory agreement with the theoretical estimates. The estimate of the vibration induced maximum electrical power output is $60 \mu \mathrm{W}$ at a matching load of $4 \mathrm{M} \Omega$ and at $\mathrm{f}=21 \mathrm{~Hz}$ and shaker acceleration of $0.02 \mathrm{~g}$. The structure based on high efficiency magnetoelectric transducers may be applied in electromagnetic energy harvester to produce enough energy for microelectronic devices.

\section{Acknowledgment}

This work was supported by the Russian Ministry of Education and Science within the framework of a State Order and RFBR research project \#13-02-98801. The authors (Y. Z. and S. P.) also thank the Office of Basic Energy Science, Department of Energy (DE-FG02-06ER46290) and Office of Naval Research for supporting the research through Center for Energy Harvesting Materials and Systems.

\section{References}

[1] M.I. Bichurin, V.M. Petrov, Advances in Condensed Matter Physics, 2012, 798310 (2012).

[2] S. Roundy and P. K. Wright, Smart Mater. Struct.13, 1131 (2004).

[3] S. Priya, D.J. Inman (Eds.), Energy Harvesting Technologies, Springer, 2009.

[4] S. Priya, R. Islam, S. X. Dong, and D. Viehland, J. Electroceram.19, 149 (2007).

[5] C. L. Zhang, J. S. Yang, and W. Q. Chen, App. Phys. Lett., 95, 013511 (2009)

[6] T.-D.Onuta, Yi Wang, C.J. Long, and I. Takeuchi, App. Phys. Lett., 99, 203506 (2011).

[7] S. Dong, J. Zhai, J. F. Li, D. Viehland, and S. Priya, App. Phys. Lett., 93, 103511 (2008)

[8] Z. Yang, J.W. Zu, L. Guo, IEEE Trans. on Magnetics, 48, 3344 (2012).

[9] Yu. Zhou, D. J. Apo, and S. Priya, Appl. Phys. Lett., 103, 192909 (2013).

[10]S. P. Timoshenko and D. H. Young. Vibration problems in engineering. 3rd ed. N. Y.: Van Nostrand Co., Inc., 1955. 